\title{
Simulation study of electron cloud induced instabilities and emittance growth for the CERN Large Hadron Collider proton beam
}

\author{
E. Benedetto* \\ Politecnico di Torino, Italy and CERN, Geneva, Switzerland \\ D. Schulte and F. Zimmermann \\ CERN, Geneva, Switzerland \\ G. Rumolo \\ GSI, Germany \\ (Received 23 September 2004; published 21 December 2005)
}

\begin{abstract}
The electron cloud may cause transverse single-bunch instabilities of proton beams such as those in the Large Hadron Collider (LHC) and the CERN Super Proton Synchrotron (SPS). We simulate these instabilities and the consequent emittance growth with the code HEADTAIL, which models the turn-byturn interaction between the cloud and the beam. Recently some new features were added to the code, in particular, electric conducting boundary conditions at the chamber wall, transverse feedback, and variable beta functions. The sensitivity to several numerical parameters has been studied by varying the number of interaction points between the bunch and the cloud, the phase advance between them, and the number of macroparticles used to represent the protons and the electrons. We present simulation results for both LHC at injection and SPS with LHC-type beam, for different electron-cloud density levels, chromaticities, and bunch intensities. Two regimes with qualitatively different emittance growth are observed: above the threshold of the transverse mode-coupling (TMC) type of instability there is a rapid blowup of the beam, while below this threshold a slow, long-term, emittance growth remains. The rise time of the TMC instability caused by the electron cloud is compared with results obtained using an equivalent broadband resonator impedance model, demonstrating reasonable agreement.
\end{abstract}

DOI: $10.1103 /$ PhysRevSTAB.8.124402

PACS numbers: 29.27.Bd, 52.65.Rr, 52.27.Jt, 52.35.Qz

\section{INTRODUCTION}

Instabilities, beam loss, and beam-size blowup due to electron cloud have been observed in several machines, such as the CERN Proton Synchrotron (PS), the Super Proton Synchrotron (SPS), as well as the KEKB and PEP-2 B-factories [1]. Therefore, they represent a concern for the future Large Hadron Collider (LHC) at CERN. In this paper we discuss simulations of transverse singlebunch instabilities using the code HEADTAIL [2,3].

During the passage of a bunch, the electrons are accumulated around the beam center (pinch effect) and, if the head of the bunch is slightly offset, the rest of the bunch will experience a net "wake" force. The instability is similar to the regular transverse mode-coupling instability (TMCI) and induces both a centroid and a head-tail motion, with a substantial emittance growth.

HEADTAIL is a particle-in-cell (PIC) code which models the interaction of a single bunch with an electron cloud on successive turns, with the simplification that the cloud is localized at a finite number of positions along the circumference, instead of being continuously spread over the entire ring. Recently, electric conducting boundary

*Electronic address: Elena.Benedetto@cern.ch conditions have been implemented in the code [4]. They replaced the previous open-space boundaries. A description of the new boundaries will be given in Sec. II. The sensitivity of the code to numerical parameters, in particular, to the number and location of the interaction points (IPs) between the cloud and the bunch will be discussed in Sec. III. This and the following four sections show simulation results for LHC at injection. In Sec. IV we investigate the TMC-type instability and the emittance growth above the threshold as a function of the electron-cloud density, the bunch intensity and the chromaticity. Below the threshold of the strong head-tail instability there is evidence of a regime with slow emittance growth. Some preliminary studies of this phenomenon will be presented in Sec. V. We also discuss first results from an attempt to model the real lattice (Sec. VI). Specifically we have modified the code in order to represent the beta function varying around the ring, instead of considering an average value. In Sec. VII the possibility to model the electroncloud effect with a broadband impedance [5] is discussed and the results compared with the PIC simulations. Then simulations for the SPS ring with LHC-type beam are presented (Sec. VIII). Here we assume the electron cloud to be concentrated in the dipole field regions. Finally, Sec. IX summarizes the results and draws an outline for future work and development. 


\section{HEADTAIL CODE AND THE NEW CONDUCTING BOUNDARY CONDITIONS}

The code HEADTAIL for the single-bunch instability has been described in Refs. [6,7]. The simulation models the turn-by-turn interaction of a single bunch with an electron cloud, which is assumed to be produced by the preceding bunches and is usually taken to be initially uniform. Its density is inferred from parallel simulations with the ECLOUD code [8]. For the purpose of the simulation, the electron cloud is assumed to be concentrated at one or more interaction points around the ring and a fresh uniform electron distribution is created at each IP prior to each bunch passage. Both the protons and the electrons are represented by macroparticles. The bunch is also divided into longitudinal slices which interact with the cloud on successive time steps. The principle of the simulation is schematically illustrated in Fig. 1.

The transverse electric interaction between the electrons and the protons of each slice (and vice versa) is computed by a 2D PIC module taken from a beam-beam code [9]. In between, the beam is transported around the ring, where the betatron motion in both planes is modeled by a rotation matrix. The synchrotron motion is included, so that the particles slowly mix longitudinally. In particular, they can move from one bunch slice to another during several turns. The effect of chromaticity is also modeled, via an additional rotation matrix. In the code there is the further possibility to include space charge and the effect of a broadband resonator. Feedbacks and various nonlinear fields are optionally available as well.

Recently new boundary conditions of a perfectly conducting chamber wall have been implemented, as an alternative to the previously applied open-space conditions. With conducting boundaries, the electric potential is assumed to be zero on the wall. A fast-Fourier-transform Poisson solver for a rectangular pipe, based on sine transformations, is used. The electric field can significantly differ from the open-space case especially in the proximity of the boundary wall.

Theoretical ratios of the horizontal electric field computed for open-space and conducting boundaries for a beam centered in a rectangular chamber of half-width $a$ and half-height $b$, at the wall $(x=a, y=0)$ are expressed

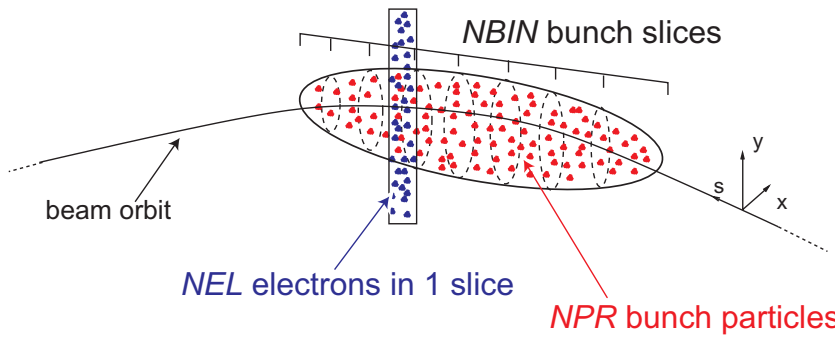

FIG. 1. (Color) Schematic of the physical model for the cloudbeam interaction in the HEADTAIL code. through the analytical formula [4], obtained by summing the contributions from the source and image charges:

$$
\begin{aligned}
\frac{E_{b c}}{E_{\text {os }}}= & 2 \sum_{m=0}^{\infty} \frac{(-1)^{m}}{2 m+1}+4 \sum_{m=0}^{\infty} \frac{(-1)^{m}}{2 m+1} \\
& \times \sum_{n=1}^{\infty} \sin ^{2}\left[\operatorname{arctg}\left(\frac{2 m+1}{2 n} \frac{a}{b}\right)\right](-1)^{n},
\end{aligned}
$$

which results in

$$
\left.\frac{E_{b c}}{E_{o s}}\right|_{a=b}=\left.1.31 \quad \frac{E_{b c}}{E_{o s}}\right|_{a=2 b}=0.54
$$

This theoretical ratio is very satisfactorily reproduced by our Poisson solver.

The difference between the electric field in open space and in a rectangular box becomes more critical as we move closer to the box wall in both directions. Figure 2 shows the vertical components of the electric field on a line
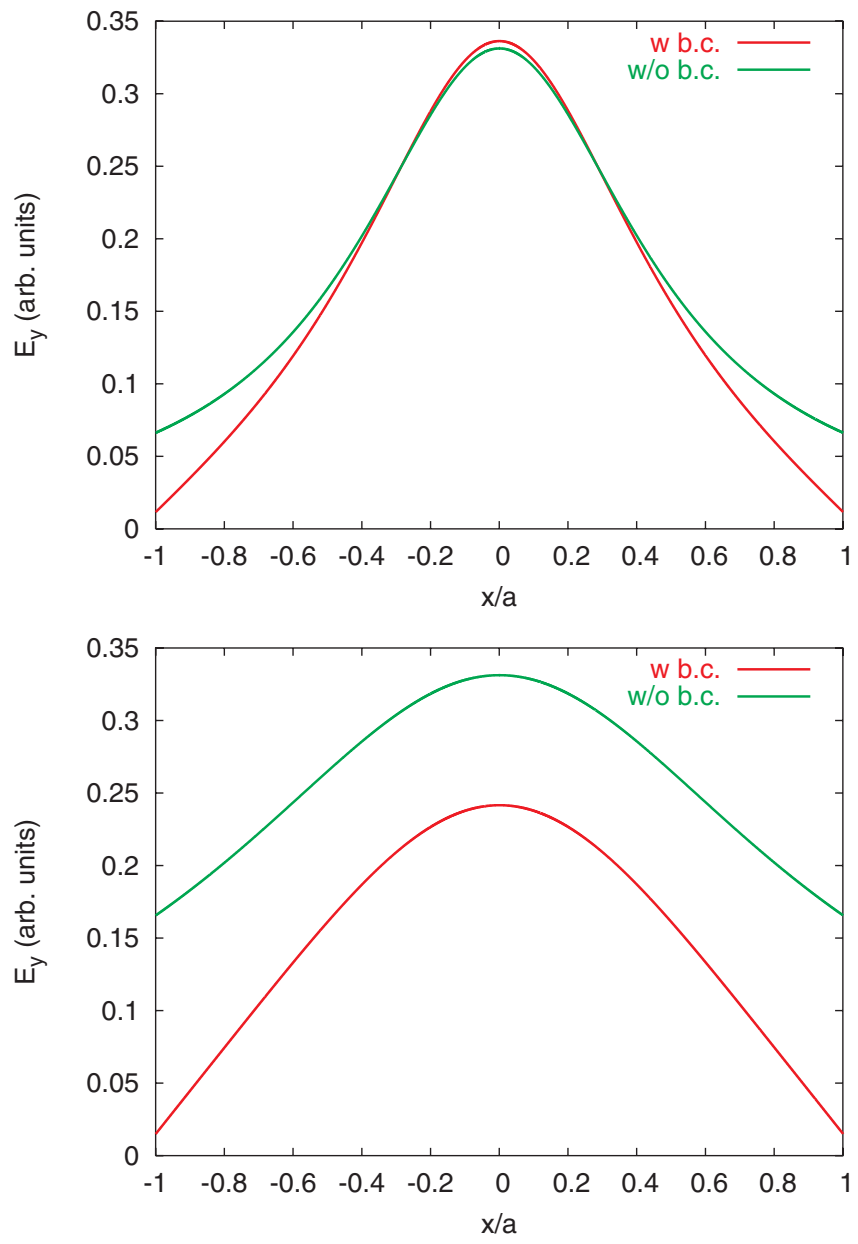

FIG. 2. (Color) Vertical electric field as a function of the horizontal position along the axis $y=+b / 2$ of a square (top) and of a rectangular chamber with $a=2 b$ (bottom), computed with and without conducting boundary conditions, for a beam centered in the chamber, with a transverse rms size $\sigma_{b}=a / 10$. 
$y=+b / 2$. The fields differ in this region by more than a factor 2 .

\section{SENSITIVITY TO NUMERICAL PARAMETERS}

For the purpose of checking the sensitivity to numerical parameters we have performed a series of simulations for the LHC at injection, assuming a typical electron-cloud density of $6 \times 10^{11} \mathrm{~m}^{-3}[10]$. Throughout this paper, if not stated otherwise, we use the bunch and numerical parameters listed in Tables I and II. In Fig. 3 we show the vertical emittance as a function of time for different numbers of electron macroparticles. A number of $10^{5}$ macroelectrons at every IP was chosen in the following. If the cloud is initialized with a transversely uniform distribution inside the chamber, this value corresponds to about 6.1 macroparticles per cell (the number of grid points over $\pm 10 \sigma$ is 128). The number of macroprotons is taken to be $3 \times 10^{5}$ and the bunch is divided into 70 slices, in order to resolve the transverse wakefield. Since during the passage of a bunch the electrons perform about 4 oscillations [11], this number of slices translates into about 17 time steps per oscillation period.

A key parameter which needs to be set carefully in the simulations is the number of beam-cloud interaction points per turn. The sensitivity to this parameter was first pointed out by Ohmi $[12,13]$. Figure 4 shows the horizontal and vertical emittance as a function of time for different numbers of IPs per turn. In the vertical plane there is clear evidence of a different behavior for a small number of IPs. Looking at the snapshot of the vertical bunch shape (Fig. 5) in the case of only 1 point of interaction per turn, the emittance growth appears incoherent and it occurs almost uniformly along the entire bunch, while in the case of 5 IPs

TABLE I. Parameters used for LHC at injection.

\begin{tabular}{lcc}
\hline \hline Electron-cloud density & $\rho_{e}$ & $6 \times 10^{11} \mathrm{~m}^{-3}$ \\
Bunch population & $N_{b}$ & $1.1 \times 10^{11}$ \\
Beta function & $\beta_{x, y}$ & $100 \mathrm{~m}$ \\
rms bunch length & $\sigma_{z}$ & $0.115 \mathrm{~m}$ \\
rms beam size & $\sigma_{x, y}$ & $0.884 \mathrm{~mm}$ \\
rms momentum spread & $\delta_{\text {rms }}$ & $4.68 \times 10^{-4}$ \\
Synchrotron tune & $Q_{s}$ & 0.0059 \\
Momentum compaction fact & $\alpha_{c}$ & $3.47 \times 10^{-4}$ \\
Circumference & $C$ & $26.659 \mathrm{~m}$ \\
Nominal tunes & $Q_{x, y}$ & $64.28,59.31$ \\
Chromaticity & $Q_{x, y}^{\prime}$ & 2,2 \\
Space charge & & $\mathrm{No}$ \\
Magnetic field & & $\mathrm{No}$ \\
Linear coupling & & $\mathrm{No}$ \\
Average dispersion & $D$ & $0 \mathrm{~m}$ \\
Relativistic factor & $\gamma$ & 479.6 \\
Particle momentum & $p$ & $850 \mathrm{GeV} / c$ \\
Cavity voltage & $V$ & 35640 \\
Cavity harmonic number & $h$ & \\
\hline \hline
\end{tabular}

TABLE II. Computational parameters.

\begin{tabular}{lcc}
\hline \hline \# of macroelectrons & NEL & $10^{5}$ \\
\# of macroprotons & $N P R$ & $3 \times 10^{5}$ \\
\# of slices & $N B I N$ & 70 \\
\# of grid points & $N$ & $128 \times 128$ \\
Size of the grid & $\sigma_{g}$ & $10 \sigma_{x, y}$ \\
Extension of the bunch in $z$ & & $\pm 2 \sigma_{z}$ \\
\# of interaction points & nkick & 10 \\
\hline \hline
\end{tabular}

the growth is due to the strong head-tail instability. Hence, for the set of parameters listed in Table I, a number of IPs larger than 5 is required to capture the physics of the instability in the case of LHC at injection energy; in our simulations we have chosen nkick $=10$.

The location of the points of interaction along the ring and the phase advance between them is also important. In the code, the IPs are normally equally spaced, their position is fixed along the ring and does not change from turn to turn. Simulations were also performed for a random phase advance between IPs, where only the total number of IPs over the circumference is given, but their location and phase advance along the ring are chosen randomly on every turn. Figure 6 shows that in this case for a small number of IPs the growth is larger than for a constant phase advance and that the convergence is very poor, but the change is monotonic and there is no evidence of two different types of behavior. The larger growth is probably due to additional noise introduced by the random choice of phase advance leading to a permanent small mismatch.

We have also tried to consider IPs whose positions were chosen randomly (instead of uniform spacing) but stayed constant from turn to turn, or to concentrate IPs over one betatron wavelength only [14], but in neither case did we observe an improvement of convergence for smaller num-

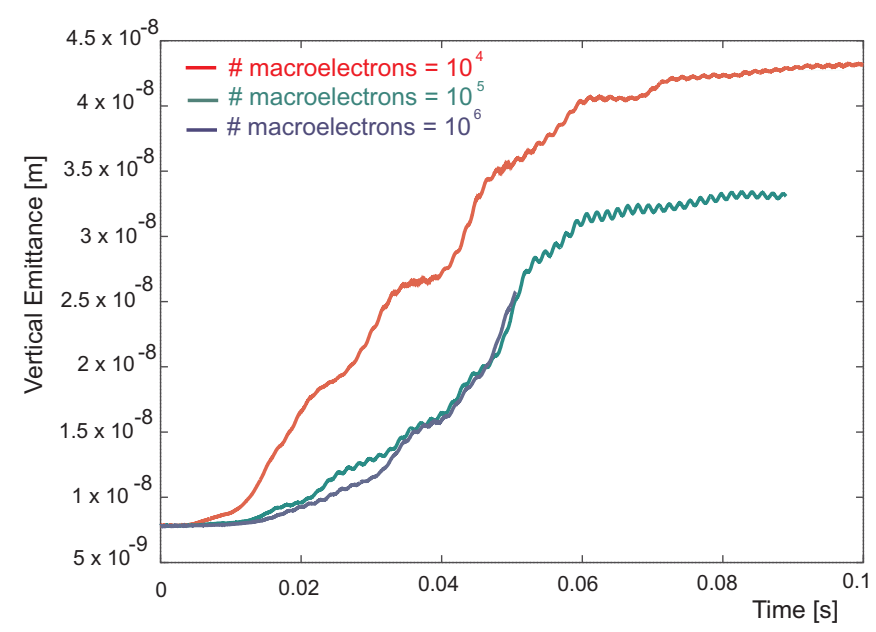

FIG. 3. (Color) Emittance as a function of time for different numbers of macroelectrons. 

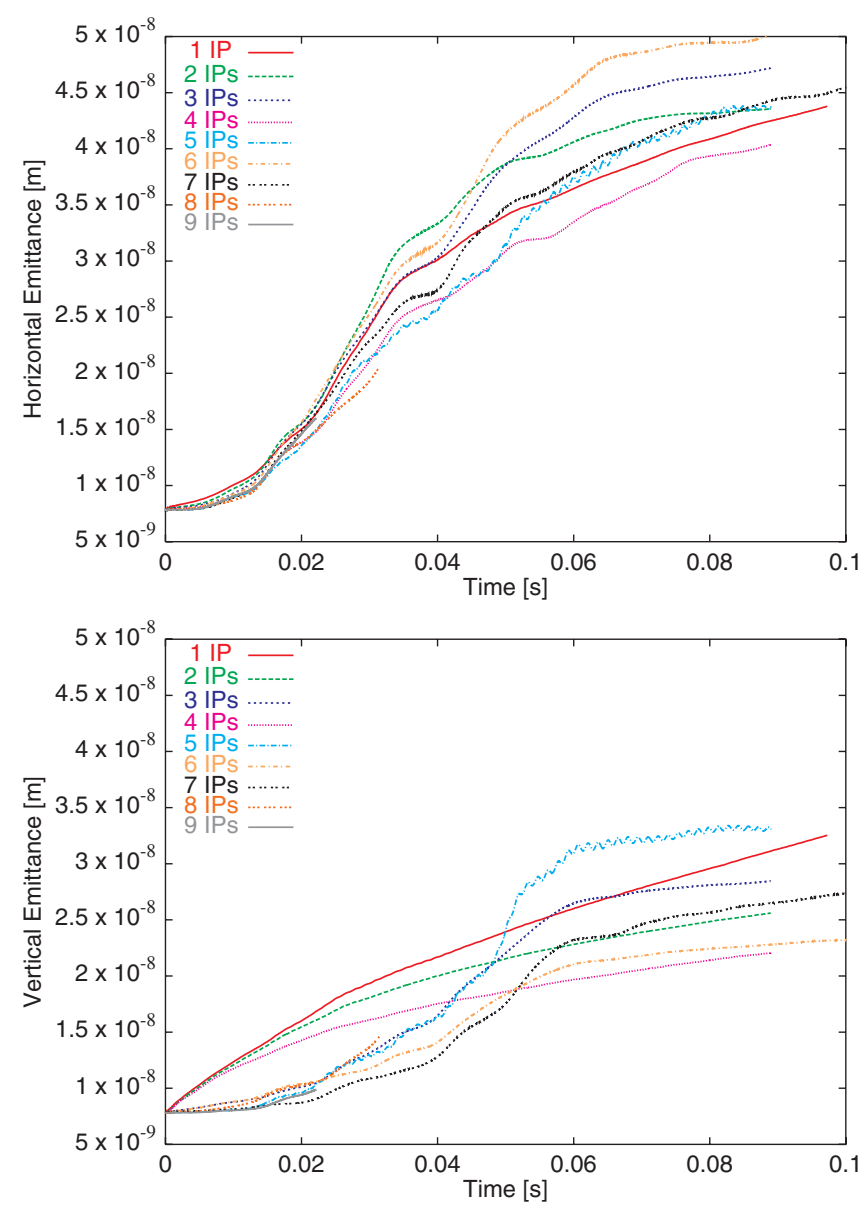

FIG. 4. (Color) Horizontal (top) and vertical (bottom) emittance as a function of time with different numbers of IPs, for the LHC at injection and an electron density $\rho=6 \times 10^{11} \mathrm{~m}^{-3}$.

ber of IPs. Moreover the emittance-growth level was similar to the one obtained with equally spaced IPs.

The effect of the distribution of rf cavities and regions with nonzero momentum compaction between the points

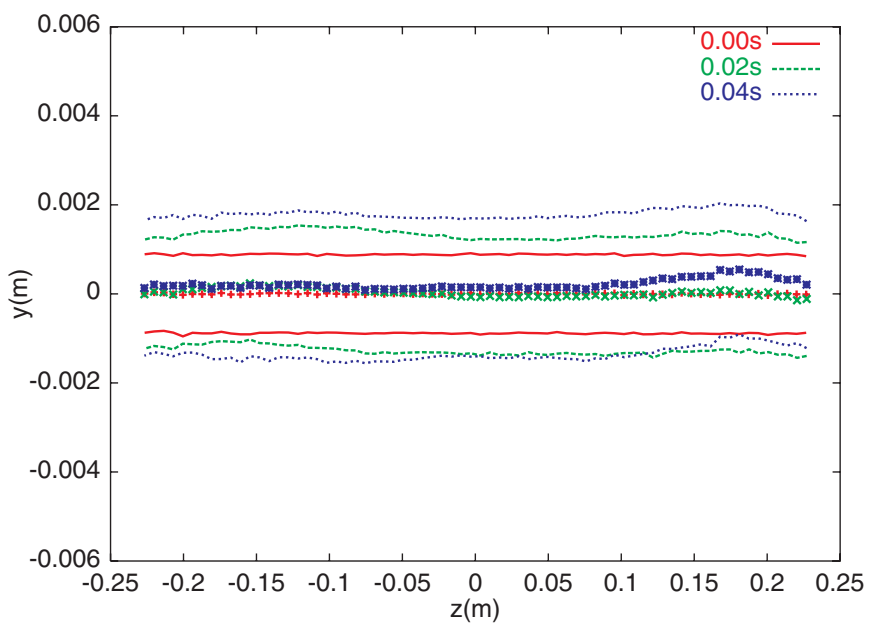

of interactions has also been studied as a possible source of discrepancies for different numbers of IPs [15], but it was found to be insignificant, at least in the simulation for the LHC.

\section{INSTABILITY THRESHOLD AND EMITTANCE GROWTH IN LHC AT INJECTION}

Using the parameters listed in Table I, we studied the effect of chromaticity, electron-cloud density, and bunch intensity on the development of the instability, again for the LHC at injection.

We first performed a scan of the electron-cloud density level in the chamber, over a range from $3 \times 10^{12} \mathrm{~m}^{-3}$ down to $2 \times 10^{11} \mathrm{~m}^{-3}$. Figure 7 shows that for $\rho=3 \times$ $10^{11} \mathrm{~m}^{-3}$ only a very small slow emittance growth remains. This value is roughly consistent with the threshold predicted by the analytical 2-particle model for the TMCI type instability [16]

$$
\rho_{\text {thre }}=\frac{2 \gamma Q_{s}}{\pi r_{p} C \beta_{x, y}}
$$

which amounts to $\rho=4.3 \times 10^{11} \mathrm{~m}^{-3}$, for these parameters, and it is similar to threshold values estimated for the KEK B-factory [16-18] and for the CERN SPS [5]. For the LHC at injection, the same threshold density of $3 \times$ $10^{11} \mathrm{~m}^{-3}$ was first determined from simulations in [19].

Figure 8 displays the emittance-growth rise time as a function of the electron-cloud density on a logarithmic scale. This figure suggests that though the emittance growth decreases for smaller electron-cloud densities, it never fully vanishes. Emittance growth on a longer time scale therefore is a concern even for moderate or low electron densities.

In Fig. 9 a scan of the bunch intensity, for an electron cloud of $6 \times 10^{11} \mathrm{~m}^{-3}$ and low chromaticity, shows that at half the nominal bunch intensity we are below the thresh-

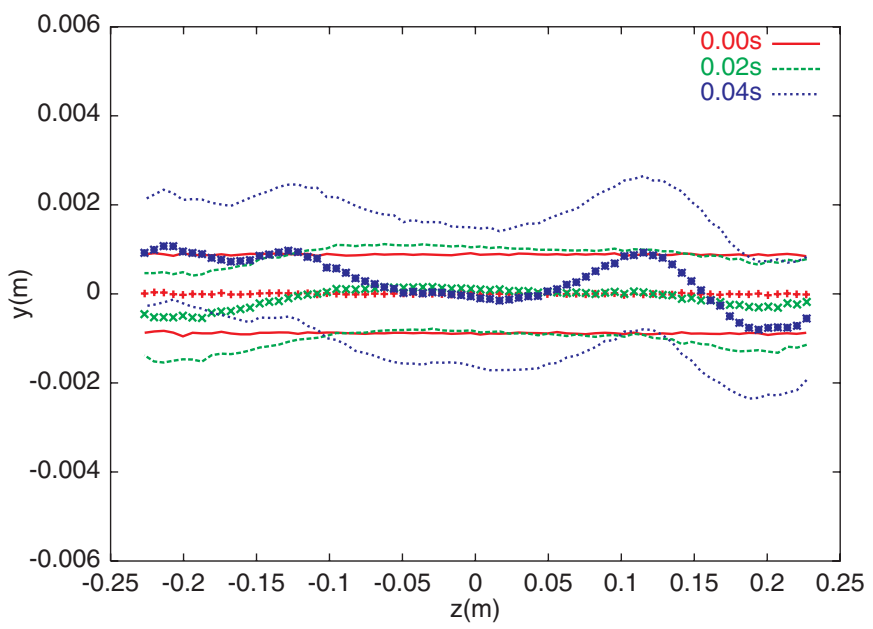

FIG. 5. (Color) Snapshot of the vertical bunch shape (centroid and rms size) at different time steps assuming 1 IP (left) and 5 IPs (right) per turn. 

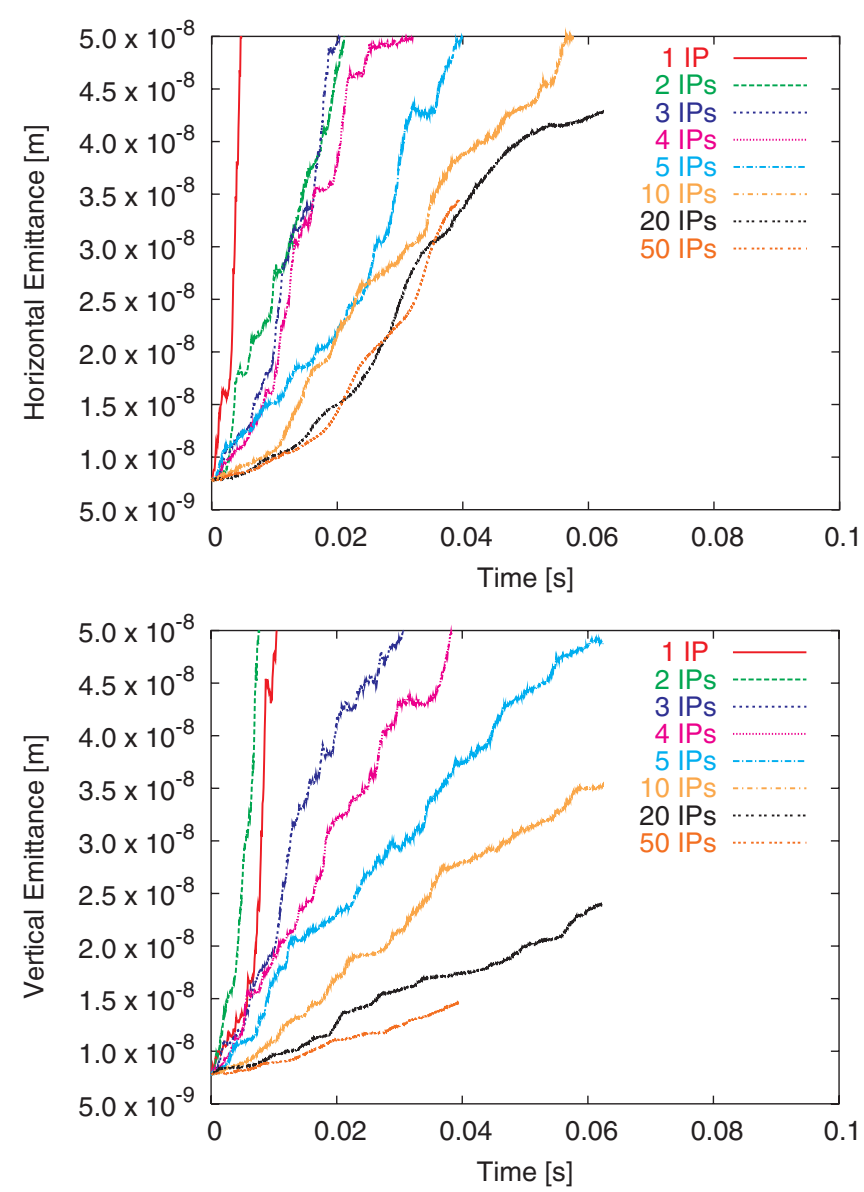

FIG. 6. (Color) Emittance growth for a turn-by-turn random phase advance between IPs; horizontal (top) and vertical (bottom) emittance as a function of time with different numbers of IPs, for LHC at injection and an electron density of $\rho=6 \times$ $10^{11} \mathrm{~m}^{-3}$.

old of the strong head-tail instability, and, at least for the first $50 \mathrm{~ms}$, the emittance growth is strongly reduced.

Assuming an electron-cloud density of $6 \times 10^{11} \mathrm{~m}^{-3}$, at nominal bunch intensity, increasing the chromaticity helps

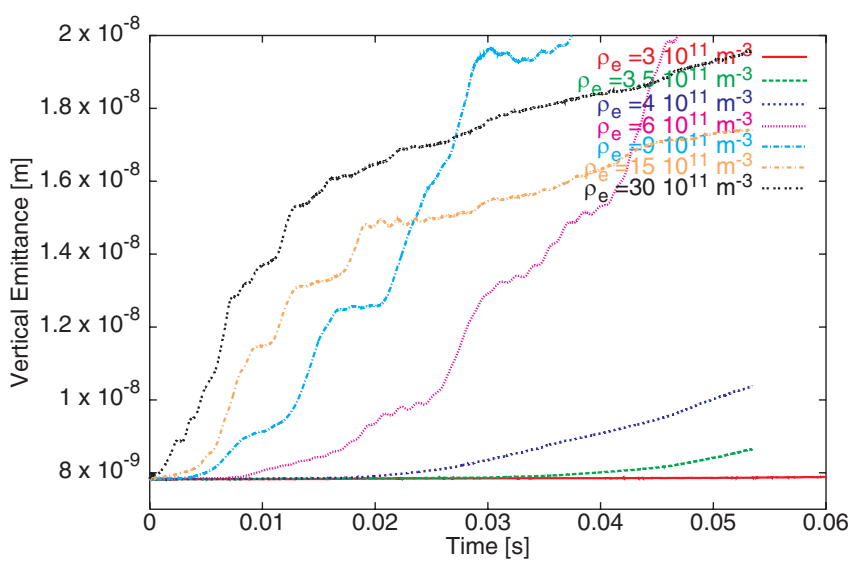

FIG. 7. (Color) Vertical emittance as a function of time for different values of electron-cloud density, and $Q^{\prime}=2$.

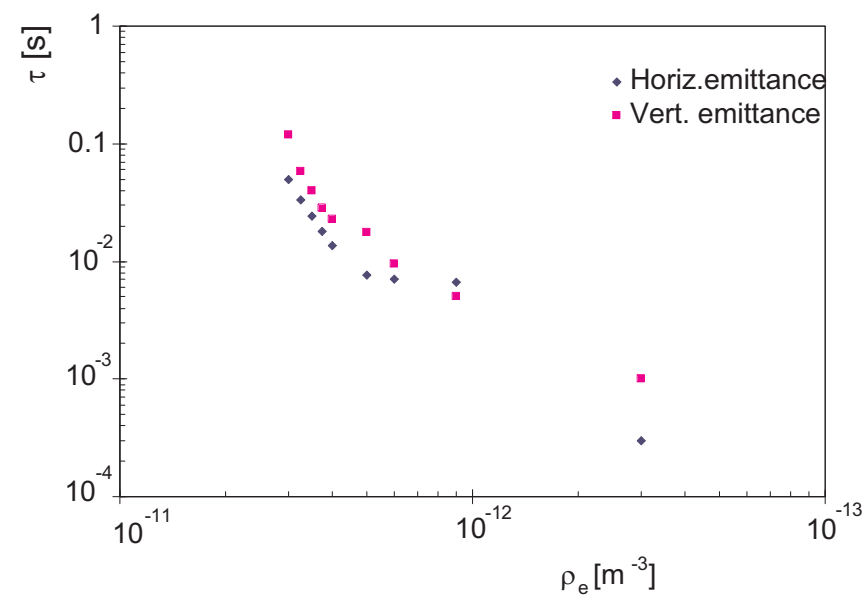

FIG. 8. (Color) Rise time $\tau$ as a function of the electron-cloud density; the rise time $\tau$ is defined as the time corresponding to an emittance increase from $7.82 \times 10^{-9} \mathrm{~m}$ (initial value) to $8 \times$ $10^{-9} \mathrm{~m}(+2.3 \%)$, at $Q^{\prime}=2$.

to reduce the emittance growth (Fig. 10), until for very high values of $Q^{\prime}=30$ we enter into a second regime, without a rapid instability, but with a persistent slow emittance growth. The threshold value of chromaticity for which the strong head-tail instability is suppressed depends on the electron-cloud density. The relation found in our simulations (see Fig. 11) is almost linear, as predicted by analytical computations for the TMC instability due to a broadband-resonator model [20]. As indicated by Fig. 11, the second regime with slow emittance growth extends down to low electron densities and it can be found, below the TMCI threshold, even for zero chromaticity.

\section{V. "SLOW EMITTANCE GROWTH" REGIME}

A simulation campaign is ongoing to understand whether the persistent slow emittance growth which we

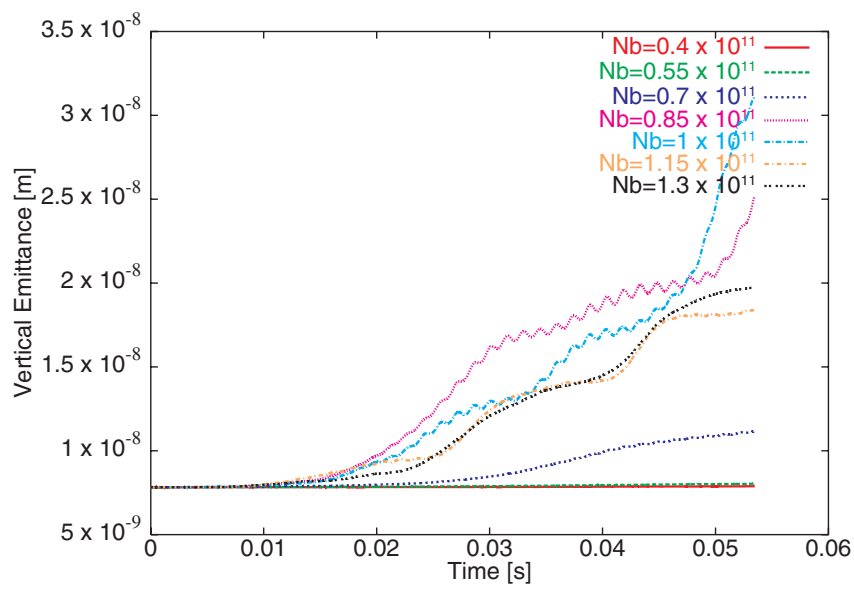

FIG. 9. (Color) Vertical emittance as a function of time for different values of bunch intensities, for LHC at injection, $\rho=$ $6 \times 10^{11} \mathrm{~m}^{-3}$, and $Q^{\prime}=2$. 


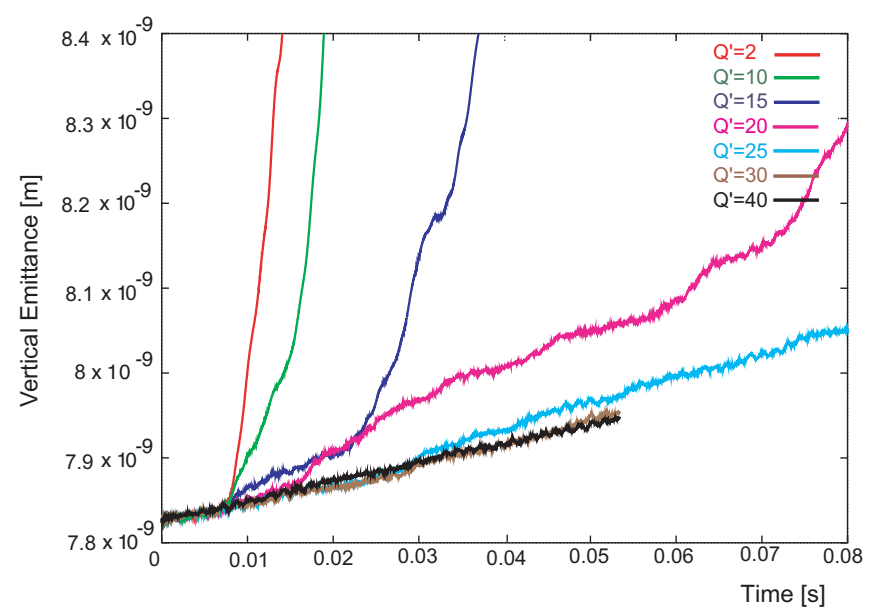

FIG. 10. (Color) Vertical emittance growth for different chromaticities, at $\rho_{e}=6 \times 10^{11} \mathrm{~m}^{-3}$.

found below the threshold is real or an artifact of the code. We note similar growth has been observed in some measurements at the KEK B-factory [21]. Preliminary results show that increasing the number of macroprotons (NPR) helps reducing this linear growth. However, the growth does not seem to approach zero in the limit of very large NPR, as illustrated in Fig. 12, which shows the dependence on $1 /$ NPR.

Changing the longitudinal bunch extent in the simulations from $\pm 2 \sigma_{z}$ to $\pm 4 \sigma_{z}$, together with the number of slices, seems to modify the behavior. Figure 13 shows that considering $\pm 4 \sigma_{z}$, of a Gaussian bunch while keeping the number of macroprotons constant causes some artificial instability, probably due to the small number of macroprotons in the tails, which may introduce a large numerical noise.

Finally, simulations have also been done for electroncloud densities below the threshold of the fast (strong

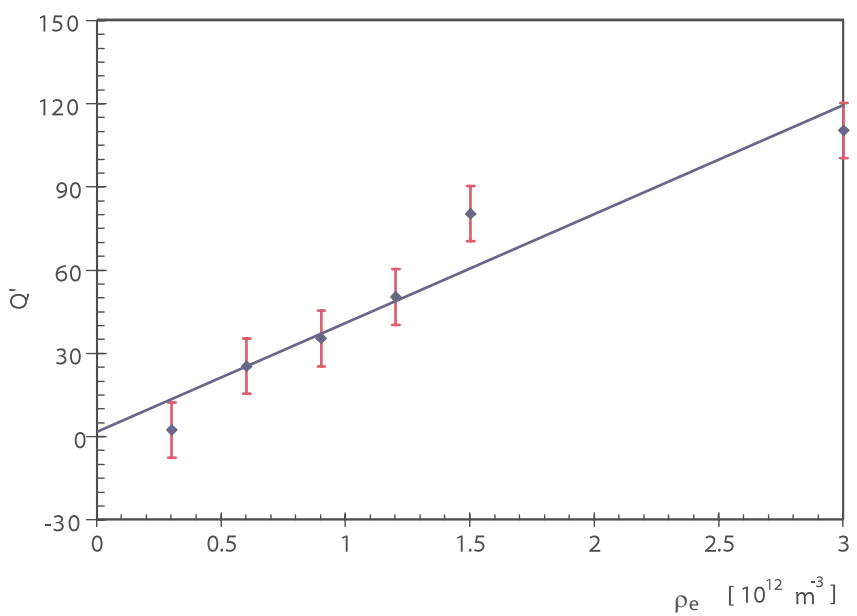

FIG. 11. (Color) Chromaticity as a function of the electroncloud density level at which the transition between the two regimes occurs in the simulation.

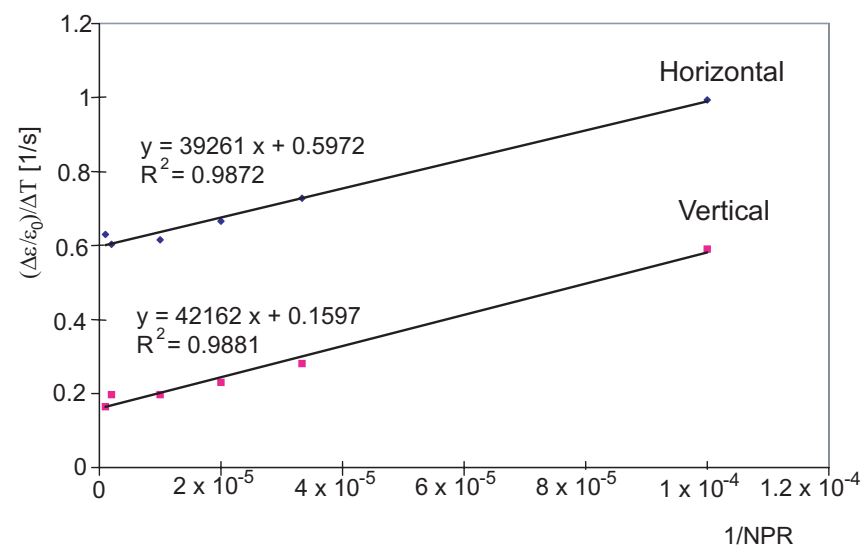

FIG. 12. (Color) Horizontal and vertical emittance-growth rate as a function of the inverse of the number of macroprotons (1/NPR), in the "slow" emittance-growth regime; the electroncloud density is $\rho_{e}=6 \times 10^{11} \mathrm{~m}^{-3}$ and the chromaticity $Q^{\prime}=$ 40 .

head-tail) instability, at different values of chromaticity (see Fig. 14). The rise time in this slow growth regime depends on the electron-cloud density via a power law

$$
\frac{1}{\epsilon_{0}} \frac{\Delta \epsilon}{\Delta t} \sim \rho_{e}^{a} \quad \text { where } a \approx 1.6-1.7,
$$

with only a weak dependence on the chromaticity.

\section{BETA FUNCTION}

In the original HEADTAIL code and in the simulations presented so far, the beta function was assumed to be constant over the whole ring and equal to the average value. Recent modifications allow us to consider different values of $\beta$ at the different IPs, thus crudely modeling the effect of the variation of the beta function around the ring (pictures of the LHC optics can be found in Ref. [10]). Our

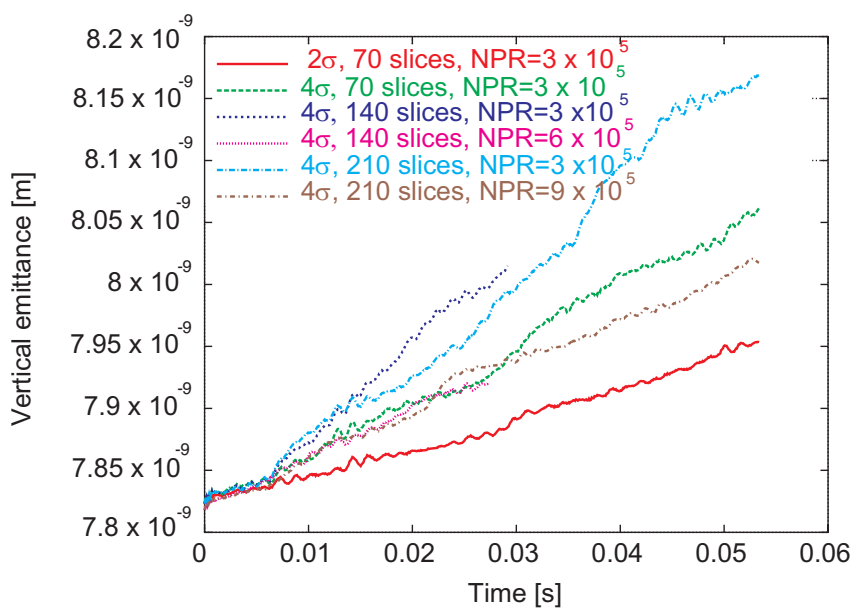

FIG. 13. (Color) Vertical emittance as a function of time, for varying longitudinal bunch extent, number of slices and number of macroprotons. 


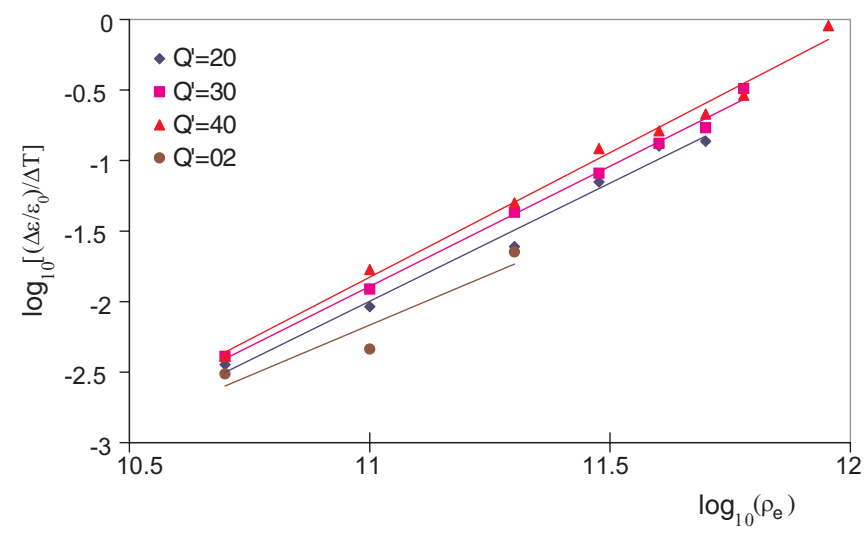

FIG. 14. (Color) Double logarithmic plot of the vertical emittance-growth rate as a function of the cloud density, for different chromaticities.

approximate treatment considers the spread in the beam sizes and the corresponding distribution in the electron oscillation frequency close to the beam, but it does not attempt to represent the real LHC optics with 64 or 59 horizontal and vertical betatron oscillations per turn. Figure 15 shows the effect in the simulations, comparing different cases, both above the fast instability threshold $\left(\rho_{e}=6 \times 10^{11} \mathrm{~m}^{-3}\right)$ and below $\left(\rho_{e}=3 \times 10^{11} \mathrm{~m}^{-3}\right)$. Above the threshold, for 3 IPs the beta-function variation affects the results, especially when it is large, but, as already shown in the previous paragraphs, with a small number of IPs the simulations are not accurate. Using 10 IPs, with different $\beta$ patterns, the curves differ only slightly. In particular, it seems that changing the value of $\beta$ at different locations makes the curves smoother.

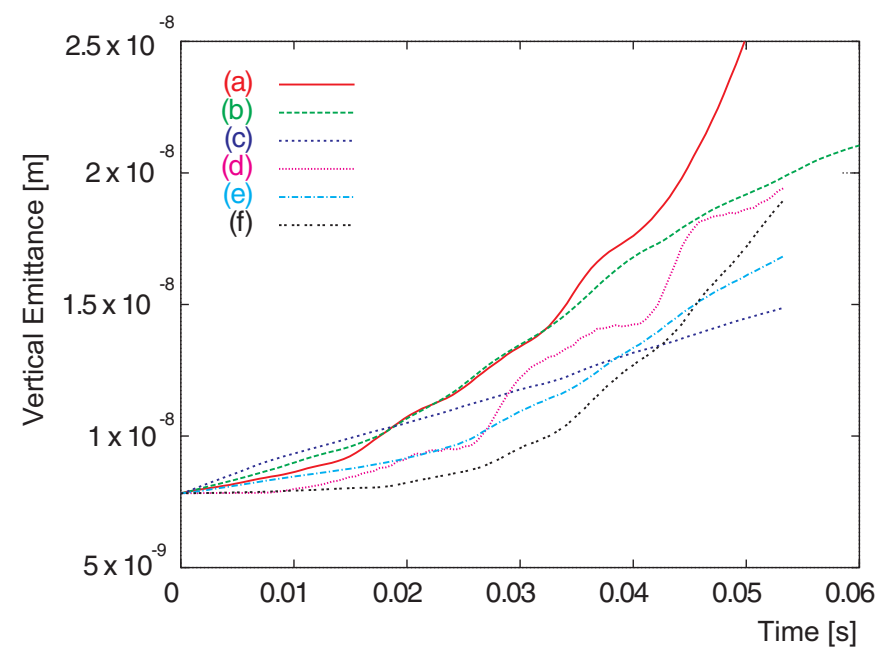

In the case below density threshold $\left(3 \times 10^{11} \mathrm{~m}^{-3}\right.$, right picture), the growth rate is modified for high numbers of IPs and is larger when we consider the variation of the beta functions along the ring. Just like diffusion introduced by space charge in intense beams because of beta modulation [22], the increase of the growth rate can be in this specific case a physical effect and its convergence for different sets of numerical parameters needs therefore further investigation. A collaboration between CERN and the University of Southern California (USC) plans to investigate the effect of the real lattice with the code QUICKPIC [23,24], which thanks to its parallel capacities allows the use of more than 2000 IPs per turn.

\section{BROADBAND IMPEDANCE MODEL FOR THE ELECTRON CLOUD}

The electron-cloud transverse wakefield responsible for single-bunch instabilities can be approximated by the one of a broadband resonator [5]

$$
W_{x, y}(z)=\left(\frac{c R_{s}}{Q}\right) \frac{\omega_{r}}{\bar{\omega}} \exp \left(\frac{\alpha}{c} z\right) \sin \left(\frac{\bar{\omega}}{c} z\right)
$$

with

$$
\begin{aligned}
\omega_{r} & =\sqrt{\frac{2 r_{e} c^{2}}{\sigma_{x, y}\left(\sigma_{x}+\sigma_{y}\right)}} \sqrt{\frac{N_{b}}{\sqrt{2 \pi} \sigma_{z}}} \frac{1}{\sqrt{k}}, \\
\left(\frac{c R_{s}}{Q}\right) & =H_{\mathrm{enh}} \frac{2^{3 / 2} \lambda_{c} r_{e}^{1 / 2} C}{\sigma_{x, y}^{3 / 2}\left(\sigma_{x}+\sigma_{y}\right)^{3 / 2} k^{3 / 2} \sqrt{\frac{N_{b}}{\sqrt{2 \pi} \sigma_{z}}}},
\end{aligned}
$$

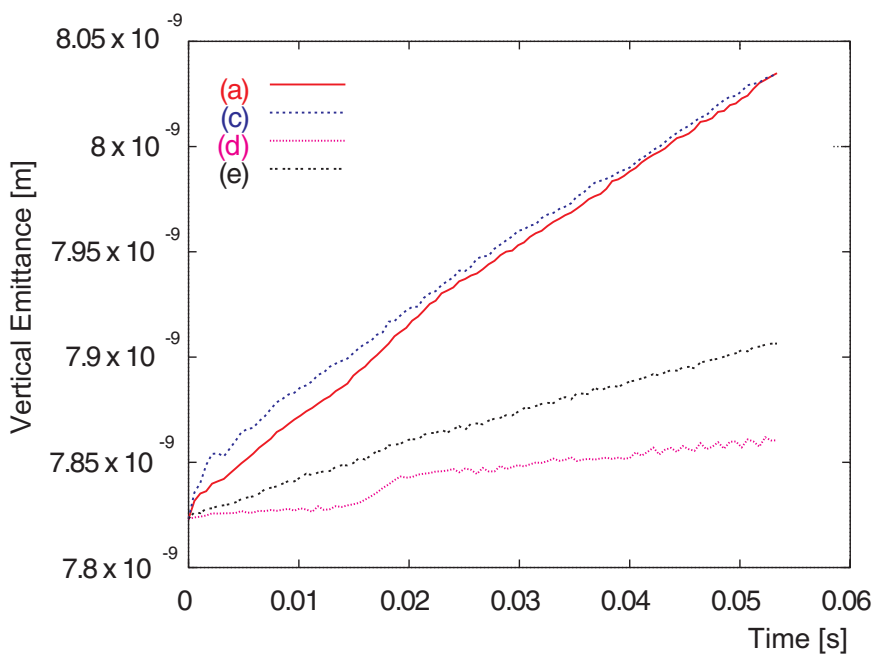

FIG. 15. (Color) Effect of considering different beta values. Vertical emittance as a function of time, with parameters of LHC at injection and chromaticity $Q^{\prime}=2$, for $\rho_{e}=6 \times 10^{11} \mathrm{~m}^{-3}$ (left) and $\rho_{e}=3 \times 10^{11} \mathrm{~m}^{-3}$ (right). Line (a) refer to simulation with 3 IPs, $\beta=100 \mathrm{~m}$ in each IP, (b) is $3 \mathrm{IPs}, \beta_{y}=100,40,160 \mathrm{~m}$, (c) is 3 IPs with $\beta_{y}=100,20,180 \mathrm{~m}$, (d) is $10 \mathrm{IPs}$, same $\beta=100 \mathrm{~m}$ over the entire ring, (e) is 10 IPs, $\beta_{y}=100,20,180,100,180,100,20,100,20,180 \mathrm{~m}$, and (f) is 10 IPs but with a different configuration of $\beta_{y}=100,100,100,180,180,180,100,20,20,20 \mathrm{~m}$. 
where $\alpha=\omega_{r} / 2 Q$ and $\bar{\omega}=\sqrt{\omega_{r}^{2}-\alpha^{2}}$. The longitudinal coordinate $z$, assuming negative values, refers to the position of the test charge with respect the driving charge. $Q$ is the quality factor, $\lambda_{c}$ the cloud line density, $c$ the light velocity, $k$ a coupling parameter, taken to be equal to 2 , and $H_{\text {enh }}$ is an enhancement factor due to the cloud size and the pinching of the electrons during the bunch passage. The quality factor $Q$ has a finite value in the range 3-6, arising from the nonlinear force acting on the electrons and the resulting frequency spread. The longitudinal beam profile and the variation of the beam size around the ring (if varying beta functions are considered), both introduce additional spreads of the electron oscillation frequency, which would further lower the effective quality factor.

For the present study, aiming to understand the instabilities induced in LHC at injection energy, we have chosen $Q=3$ and $H_{\mathrm{enh}}=9$. These values were obtained by fitting the analytical formula (5) to the wakefield from a dedicated HEADTAIL simulation for $\rho_{e}=6 \times 10^{11} \mathrm{~m}^{-3}$. Figure 16 shows the simulated wakefield and analytical curves for different combinations of $Q$ and $H_{\text {enh }}$ values, with a constant product $Q \times H_{\text {enh }}$.

In the HEADTAIL code we can model the effect of a broadband resonator [3]. Given the resonant frequency and the shunt impedance, we have directly simulated the emittance growth using one of the fitted analytical resonator wakefields of Fig. 16, instead of performing an electron-cloud PIC simulation. Figure 17 shows that contrary to what is expected from a threshold calculation in coasting beam approximation [see Eq. (12) in [25]], it is not only the product $Q \times H_{\text {enh }}$ which matters for the development of the instability, but the two variables $Q$ and $H_{\text {enh }}$ enter independently.

Figures 18 and 19 compare results of electron-cloud PIC simulations for various electron densities, with those ob-

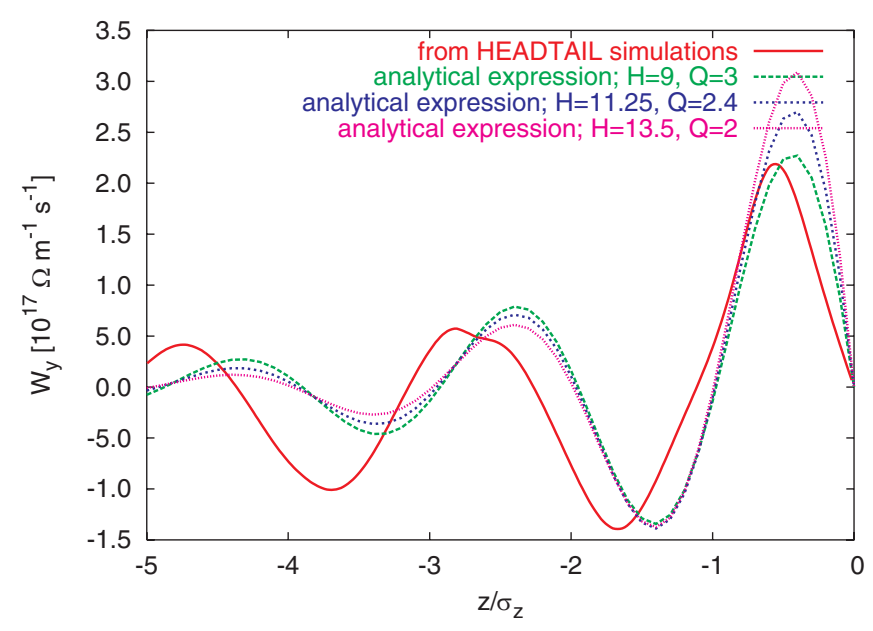

FIG. 16. (Color) Wakefield induced by an electron cloud $\left(\rho_{e}=\right.$ $\left.6 \times 10^{11} \mathrm{~m}^{-3}\right)$, in LHC at injection. The red curve is from a HEADTAIL simulation, while the other lines represent the analytical expression (5) of the wakefield.

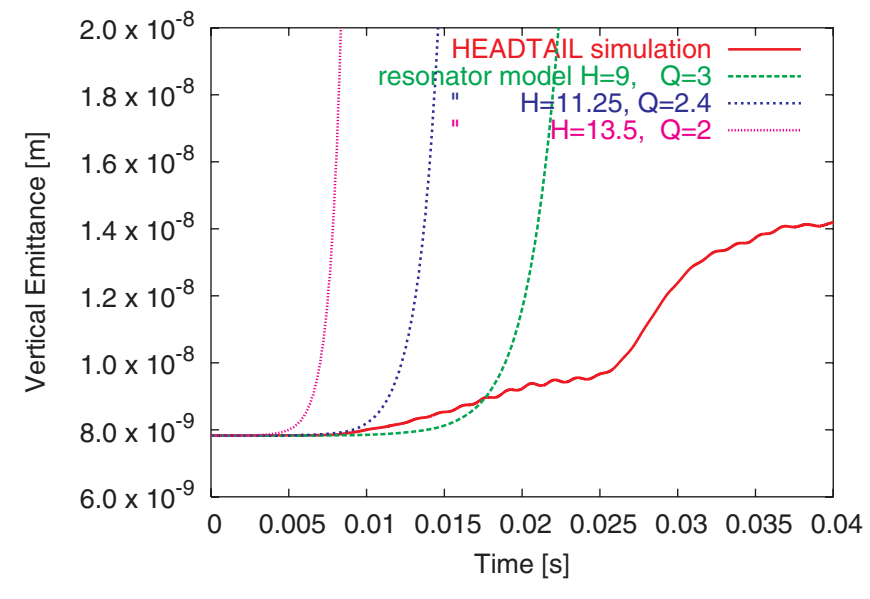

FIG. 17. (Color) Vertical emittance vs time in LHC at injection for $\rho_{e}=6 \times 10^{11} \mathrm{~m}^{-3}$ from a HEADTAIL PIC simulation (red line) and from a HEADTAIL simulation with broadband-resonator model. For the latter, different combinations of $H_{\text {enh }}$ and $Q$ are plotted, with a constant product $Q \times H_{\mathrm{enh}}$.

tained using the corresponding broadband-resonator model. The assumed correspondence, from Fig. 16, is as follows. PIC simulations for an electron cloud of $6 \times$ $10^{11} \mathrm{~m}^{-3}$ in the LHC at injection are compared with a resonator characterized by $\omega_{r}=2 \pi \times 1.199 \mathrm{GHz}, Q=$ 3 , and $Z_{t}=115.3 \mathrm{M} \Omega / \mathrm{m}$, being $Z_{t} / Q[\Omega / \mathrm{m}]=c / \omega_{r} \times$ $\left(c R_{s} / Q\right)\left[\mathrm{m}^{-2}\right] \times Z_{0} /(4 \pi)$, with $Z_{0}=377 \Omega$. For other densities, the resonator shunt impedance is varied in proportion to the change in electron density, whereas the resonator frequency, $Q$ value and enhancement factor stay constant.

Concluding this comparison, the resonator model gives initial growth rates similar to the full electron-cloud simulation over a large range of electron-cloud densities. At large amplitudes the finite size of the field grid and the nonlinear force between beam and electrons slow down the

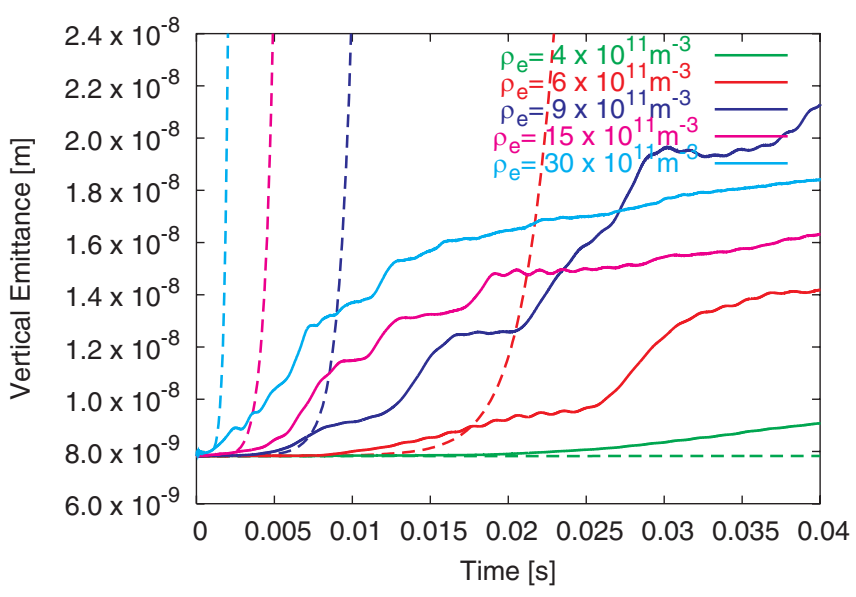

FIG. 18. (Color) Emittance growth in LHC at injection: resonator model with $H_{\text {enh }}=9$ and $Q=3$ (dotted lines) and HEADTAIL PIC module (full lines) for various electron-cloud densities. 


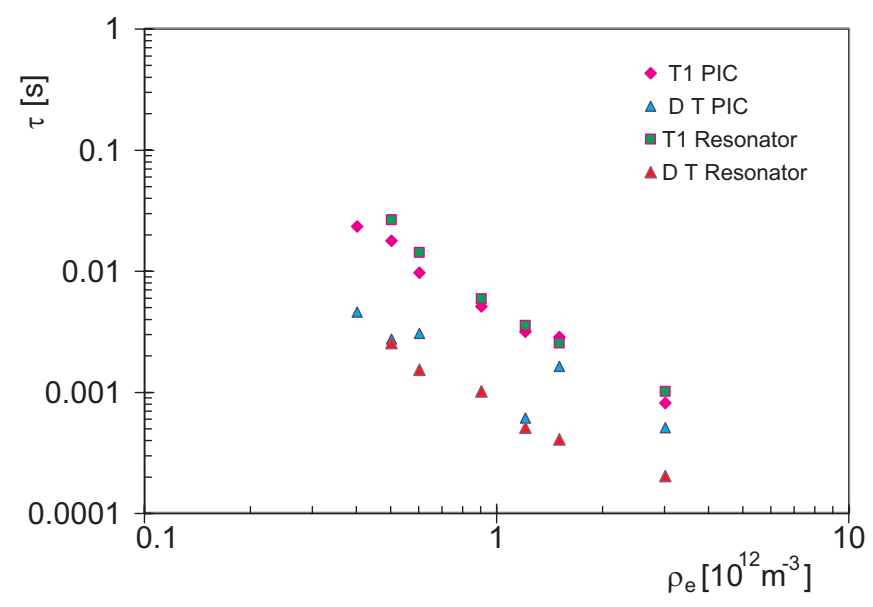

FIG. 19. (Color) Rise times of the emittance growth as a function of the electron-cloud density obtained by the HEADTAIL code with the electron-cloud PIC simulation and for an equivalent broadband resonator $\left(H_{\mathrm{enh}}=9\right.$ and $\left.Q=3\right)$; T1 denotes the time during which the emittance increases from $7.82 \times 10^{-9} \mathrm{~m}$ (initial value) to $8 \times 10^{-9} \mathrm{~m}, \Delta T$ the interval in which the emittance rises from $8 \times 10^{-9} \mathrm{~m}$ to $8.2 \times 10^{-9} \mathrm{~m}(+2.5 \%)$.

emittance growth induced by the electron cloud, in the case of the PIC calculation.

\section{HEADTAIL SIMULATION FOR SPS}

Simulations have also been performed for an LHC-type beam in SPS. The parameters of this beam are listed in Table III. The aim of these simulations is benchmarking the code against observations.

In the SPS, the electron cloud is mainly concentrated inside the bending magnets [26]. For this reason in the

TABLE III. Parameters used in the simulations for LHC-type beam in SPS at injection.

\begin{tabular}{lcc}
\hline \hline Electron-cloud density & $\rho_{e}$ & $10^{11}$ and $10^{12} \mathrm{~m}^{-3}$ \\
Bunch population & $N_{b}$ & $1.1 \times 10^{11}$ \\
Beta function & $\beta_{x, y}$ & $40 \mathrm{~m}$ \\
rms bunch length & $\sigma_{z}$ & $0.24 \mathrm{~m}$ \\
rms beam size & $\sigma_{x, y}$ & $2.1,2.1 \mathrm{~mm}$ \\
rms momentum spread & $\delta_{\text {rms }}$ & 0.002 \\
Synchrotron tune & $Q_{s}$ & 0.0059 \\
Momentum compaction fact & $\alpha_{c}$ & $1.92 \times 10^{-3}$ \\
Circumference & $C$ & $6911 \mathrm{~m}$ \\
Nominal tunes & $Q_{x, y}$ & $26.185,26.13$ \\
Chromaticity & $Q_{x, y}^{\prime}$ & $4.94,3.9$ \\
Space charge & & Optional \\
Magnetic field & & Strong field approx \\
Linear coupling & & No \\
Average dispersion & $D$ & $2.28 \mathrm{~m}$ \\
Relativistic factor & $\gamma$ & 27.728 \\
Cavity voltage & $V$ & $2 \mathrm{MV}$ \\
Cavity harmonic number & $h$ & 4620 \\
\hline \hline
\end{tabular}

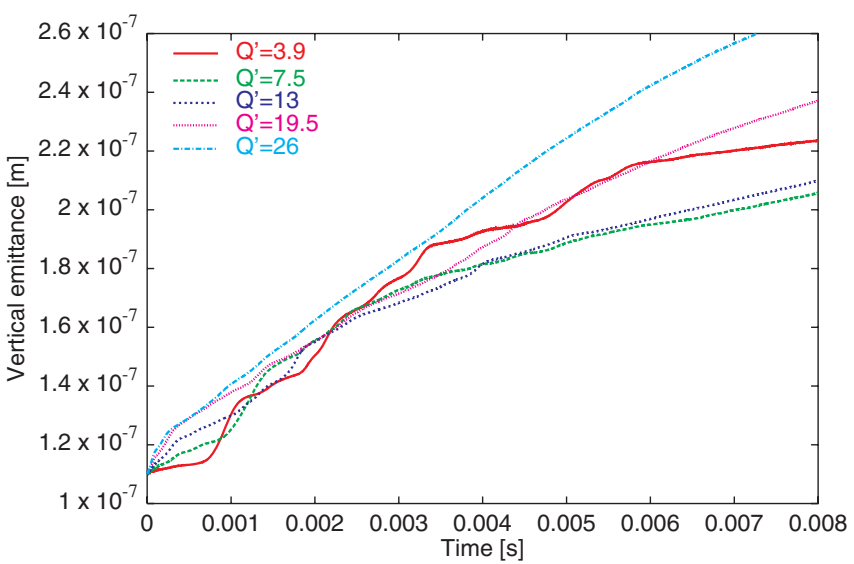

FIG. 20. (Color) Vertical emittance as a function of time for the SPS, comparing different values of vertical chromaticity $Q^{\prime}$ at $\rho=10^{12} \mathrm{~m}^{-3}$ without space charge.

simulations we have assumed the presence of a constant vertical magnetic field, which causes the electron motion to be frozen in the horizontal plane (strong field approximation). A feedback system has also been implemented in the code. It damps the transverse position of the bunch centroid, according to a specified gain. The damping time is presently assumed to be about 10 turns. The noise of the feedback system is also taken into account in the model and it is about $10^{-5} \mathrm{~m}$. The damper is found to have little effect on the single-bunch emittance growth. In fact, its main operational purpose is to cure coupled-bunch instabilities and its $20-\mathrm{MHz}$ bandwidth is too low to damp headtail motion inside a bunch.

The scan in chromaticity for an electron-cloud density of $10^{12} \mathrm{~m}^{-3}$ (Fig. 20) reveals that increasing the chromaticity only helps up to a certain value of $Q^{\prime} \approx 13$. For larger values the emittance growth increases again. Including space-charge effects in the simulation drastically changes the results (Fig. 21). Now chromaticity is much more

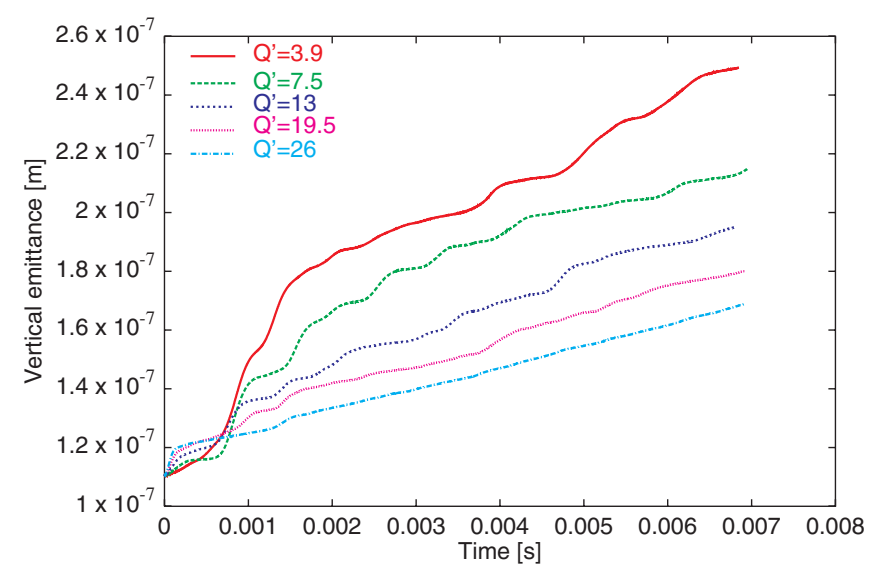

FIG. 21. (Color) Vertical emittance as a function of time for the SPS, comparing different values of vertical chromaticity $Q^{\prime}$ at $\rho=10^{12} \mathrm{~m}^{-3}$; space charge is included here. 


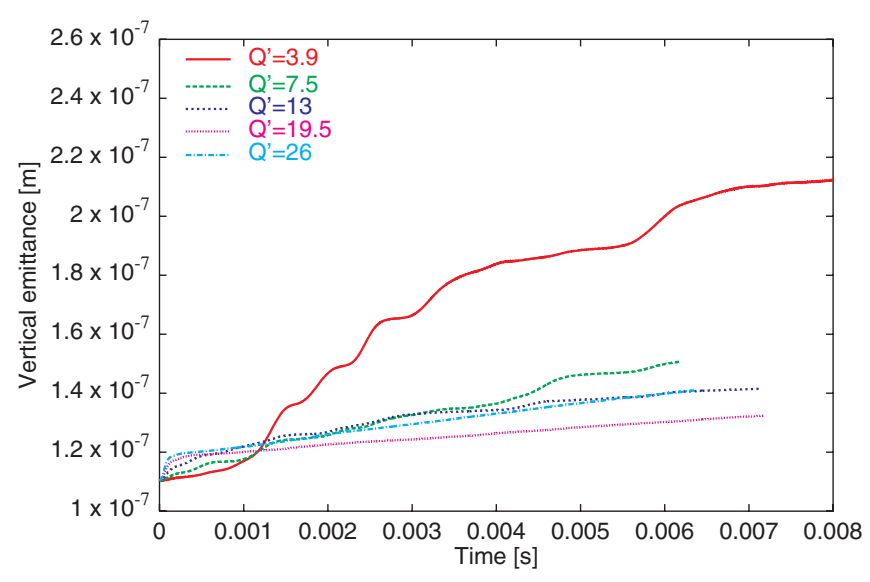

FIG. 22. (Color) Vertical emittance as a function of time for the SPS, comparing different values of vertical chromaticity $Q^{\prime}$ at $\rho=6 \times 10^{11} \mathrm{~m}^{-3}$, without space charge. A similar result was shown in Fig. 2 of [3].

efficient in damping the instability; see also [3]. Figure 22 shows that for a lower electron-cloud density $(\rho=6 \times$ $10^{11} \mathrm{~m}^{-3}$ ), even without space charge the chromaticity significantly reduces the instability growth rate.

\section{CONCLUSIONS AND OUTLOOK}

The code HEADTAIL with new conducting boundary conditions has been used to simulate single-bunch instabilities and emittance growth due to an electron cloud in the LHC and SPS rings. The sensitivity to several numerical parameters has been explored. In particular we discussed the choice of the number and position of the interaction points between the bunch and the electron cloud, which in the code are concentrated at a finite number of locations around the ring.

Simulations for LHC at injection show that chromaticity is a cure for the strong head-tail instability, but that it may not be efficient for suppressing a slow, long-term emittance growth which persists below the threshold and seems to scale with the electron density via a power law. Likely, both numerical noise and real physics contribute to this slow emittance growth. By increasing the number of macroprotons, the growth rate is reduced, but it does not approach zero in the limit of an infinite number of macroprotons. Changing the longitudinal extent of the bunch in the simulations also affects the results, but this dependence is attributed to the extremely small number of macroparticles representing the tails of the Gaussian bunch which can be a source of large numerical noise. At chromaticity $Q^{\prime}=2$ in the LHC we stay below the threshold of the TMCI type instability up to half the nominal bunch intensity for an electron density of $6 \times 10^{11} \mathrm{~m}^{-3}$. With nominal beam parameters, however, an electron density of $3 \times$ $10^{11} \mathrm{~m}^{-3}$ or less must be achieved to stay below the threshold. The dependence on chromaticity has also been studied for the SPS, where we assume the electron cloud to be concentrated in the dipole field regions. For the SPS, the space-charge effect changes the beam response to the electron cloud and renders higher chromaticity a more efficient cure.

The broadband-resonator model for the electron cloud, and the PIC simulation seem to agree at the onset of the instability for a wide range of electron densities; later the nonlinear effects, which are not taken into account in the resonator model, and the finite size of the cloud and of the grid, used for the PIC computation, become important. This leads to a different behavior at large amplitudes, which is more optimistic in the case of the real field calculation with the PIC module.

Including a variation of the $\beta$-function smoothens the evolution above the TMC threshold and changes the growth rate below the threshold, which may indicate that the long-term emittance growth seen in the latter case has a physical origin.

In the near-term future we are planning to compare SPS simulation results with ongoing experiments. Studying the behavior of the beam below the threshold of the strong head-tail instability, both via numerical and analytical approaches, is in our plans. Finally, the ongoing collaboration with USC will aim to benchmark HEADTAIL with the continuous plasma code QUICKPIC and, in more detail, investigate the effect of the real lattice on the simulation results.

\section{ACKNOWLEDGMENTS}

The authors thank G. Arduini, M. Blaskiewicz, A. Chao, A. Ghalam, S. Heifets, T. Katsouleas, E. Metral, K. Ohmi, T. Raubenheimer, and F. Ruggiero for helpful comments and discussions. We acknowledge the support of the European Community-Research Infrastructure Activity under the FP6 "Structuring the European Research Area" programme (CARE, Contract No. RII3-CT-2003506395).

[1] Proceedings of the 31st Advanced ICFA Beam Dynamics Workshop on Electron-Cloud Effects: ECLOUD04, Napa, California, 2004, edited by M. Furman, S. Henderson, and F. Zimmermann (CERN Yellow Report No. CERN-2005001, 2005).

[2] G. Rumolo and F. Zimmermann, SL-Note-2002-036,2002.

[3] G. Rumolo and F. Zimmermann, Phys. Rev. ST Accel. Beams 5, 121002 (2002).

[4] E. Benedetto, G. Rumolo, and F. Zimmermann, "HEADTAIL Manual: Updates and Modifications" (to be published).

[5] K. Ohmi, F. Zimmermann, and E. Perevedentsev, Phys. Rev. E 65, 016502 (2002).

[6] G. Rumolo and F. Zimmermann, in Proceedings of Chamonix XI, 2001 (CERN Report No. SL-2000-07). 
[7] G. Rumolo and F. Zimmermann, in Proceedings of the Particle Accelerator Conference, Chicago, IL, 2001 (IEEE, Piscataway, NJ, 2001); CERN Report No. SL2001-041, 2001.

[8] G. Rumolo and F. Zimmermann, Report No. SL-Note2002-016, 2002.

[9] D. Schulte, Ph.D. thesis, DESY/Univ. Hamburg [Report No. TESLA-97-08, 1996].

[10] CERN Report No. 2004-003, edited by O. Bruning, P. Collier, P. Lebrun, S. Myers, R. Ostojic, J. Poole, P. Proudlock, 2004.

[11] E. Benedetto and F. Zimmermann, in Proceedings of the European Particle Accelerator Conference, Lucerne, 2004 (CERN Report No. AB-2004-054, 2004).

[12] K. Ohmi, "Single-Bunch Electron-Cloud Instability for a Round Beam (memo)," http://wwwslap.cern.ch/ collective/ecloud02/ecsim/ecirb.pdf, 2002.

[13] E. Benedetto, D. Schulte, F. Zimmermann, K. Ohmi, Y. Papaphilippou, and G. Rumolo, in Proceedings of the Particle Accelerator Conference, Portland, OR, 2003 (IEEE, Piscataway, NJ, 2003).

[14] A. Chao (private communication).

[15] T. Raubenheimer and M. Pivi (private communication).

[16] K. Ohmi and F. Zimmermann, Phys. Rev. Lett. 85, 3821 (2000).
[17] K. Ohmi, in Proceedings of the Particle Accelerator Conference, Chicago, IL, 2001 (Ref. [7]).

[18] G. Rumolo, F. Zimmermann, H. Fukuma, and K. Ohmi, in Proceedings of the Particle Accelerator Conference, Chicago, IL, 2001 (Ref. [7]).

[19] E. Benedetto, D. Schulte, F. Zimmermann, and G. Rumolo, in Proceedings of Chamonix XII, 2003 (CERN Report No. AB-2003-008, 2003).

[20] E. Metral, in Proceedings of the 8th European Particle Accelerator Conference, Paris, 2002 (CERN Report No. PS 2002-022, 2002).

[21] H. Fukuma, in Proceedings of ECLOUD'04, Napa Valley, 2004 (Ref. [1]).

[22] K. Ohmi (private communication).

[23] G. Rumolo, A. Z. Ghalam, T. Katzouleas, C. Huang, V. Decyk, C. Ren, W. Mori, F. Zimmermann, and F. Ruggiero, Phys. Rev. ST Accel. Beams 6, 081002 (2003).

[24] A. Ghalam, T. Katsouleas, C. Huang, V. Decyk, W. Mori, and G. Rumolo, in Proceedings of ECLOUD'04, Napa Valley, 2004 (Ref. [1]).

[25] K. Ohmi, in Proceedings of ECLOUD'04, Napa Valley, 2004 (Ref. [1]).

[26] J. M. Jimenez, G. Arduini, P. Collier, G. Ferioli, B. Henrist, N. Hilleret, L. Jensen, K. Weiss, and F. Zimmermann, LHC Project Report No. 632, 2003. 